\title{
OBJECTIVE PRISM SPECTRAL CLASSIFICATION AT THE STOCKHOLM OBSERVATORY
}

\author{
B. NORDSTRÖM and A. SUNDMAN \\ Stockholm Observatory, Saltsjöbaden, Sweden
}

\begin{abstract}
As a result of the spectral survey at the Stockholm Observatory finding lists are prepared for early and late type stars in the Southern Milky Way. In order to make the lists more useful we present the principles of the stellar classification.
\end{abstract}

\section{Introduction}

The spectral survey of the Southern Milky Way which is continuously carried out at the Stockholm Observatory, is based on observations performed by L. O. and K. Lodén and others in the years 1956-1966 at the Boyden Observatory in South Africa. The complete observational material covers the Milky Way from $l=237^{\circ}$ to $l=7^{\circ}$ approximately between $b=+3^{\circ}$ and $b=-3^{\circ}$.

Spectral plates have been obtained with the ADH Baker-Schmidt telescope and with the Hamburg Schmidt telescope at the Boyden Observatory. The spectral plates used until now are ADH-plates with a dispersion of about $200 \AA \mathrm{mm}^{-1}$ at $\mathrm{H} \delta$. The widening is between 0.2 and $0.5 \mathrm{~mm}$. The plates cover completely a $6^{\circ}$ broad galactic band, but a considerable number of stars listed are found outside this $6^{\circ}$ band. A big portion of this survey is now finished and catalogues with spectral classification and approximative magnitudes are prepared for the regions from Puppis to Centaurus $\left(l=237^{\circ}-318^{\circ}\right)$.

These catalogues contain lists of early- and late-type stars and other objects which are considered as interesting, for example carbon and emission line stars. At the moment the catalogues exist in print (Lodén and Sundman, 1966) and preprint (Nordström, 1970; Sundman, 1970). They can be obtained from the Stockholm Observatory.

The principal aim of our classification system is to secure as much information as possible about each star from the inspection of the spectrum.

\section{The Classification Principle}

\subsection{EARLY-TYPE SPECTRA}

The early-type spectra which have been recorded at the inspection are roughly those without visible $\mathrm{K}$-line. An important exception from this rule concerns spectra with unusually narrow Balmer lines, indicating giant or supergiant A stars, interstellar $\mathrm{K}$-line, emission features, or spectral peculiarities. The main problem is the uneven quality of the material. Only on optimal exposures both spectral and luminosity classification may be performed with reasonable chance of success. We have therefore accepted the following main principles as the indication of spectral type: 
(I) If the spectrum gives no additional information, the star is considered as belonging to the main sequence. That will imply a risk that a shift upwards in the HR diagram may be interpreted as a corresponding shift towards higher temperature, i.e. a B8III may be listed as say a B5 and a B8I as even a B0. It is shown from experience, however, that this risk will cause very little disadvantage when connected with a finding list. Firstly, the main sequence stars represent a majority which becomes more and more overwhelming towards fainter apparent magnitude. Secondly, the highluminosity stars are generally highly interesting and those who use the finding list and discover the discrepancy are not likely to be disappointed. In cases when the quality permits a luminosity classification, the estimated luminosity has been indicated in the list.

(II) The spectrum showing the widest Balmer lines without visible K-line is listed as $B 9$. In a spectrum of inferior quality the K-line may be blurred out so that a systematic shift of the classification system occurs for the plate in question (if the quality drop is not an isolated phenomenon for that particular star). A corresponding shift in the opposite direction might occur for the high-quality plates on which the K-line is visible in the B9 spectra or even in the B8 spectra although this risk is rather small for an experienced classifier. Thus under extremely unfavourable circumstances (see also below) there may be a tolerance corresponding to the range B7-A2 for the spectra classified as B9 according to the standard principle of the system. The averagequality exposures are predominating, however, $(\sim 70 \%)$ and hence the tolerance is generally much smaller and conditioned by accidental phenomena (see below).

For less good plates various sources of errors become more important. Thus an interstellar K-line is not recognized when no helium or silicon lines can be seen. The star is then classified as A or later and generally omitted from the list.

The classification principles are given in Table I.

TABLE I

Classification scheme for apparent spectral types O-A0

\begin{tabular}{|c|c|c|c|}
\hline Spectral type & Optimal quality & Medium quality & Inferior quality \\
\hline $05-9$ & $\begin{array}{l}\text { According to Morgan } \\
\text { et al., } 1943\end{array}$ & Lines of HeII visible & No spectral lines visible \\
\hline O 9.5 & Only faint traces of $\mathrm{HeII}^{\mathrm{a}}$ & Lines of HeI visible & No spectral lines visible \\
\hline B 0 & $\begin{array}{l}\text { Lines of } \mathrm{He} \text { I visible. } \\
\text { Faint } \mathrm{H} \text {-lines }\end{array}$ & Faint traces of $\mathrm{H}$-lines & No spectral lines visible \\
\hline B 1 & $\begin{array}{l}\text { H-lines comparable with } \\
\text { HeI } 4026^{a}\end{array}$ & Faint traces of $\mathrm{H}$-lines & No spectral lines visible \\
\hline B 2 & $\begin{array}{l}\text { H-lines stronger than } \\
\text { HeI } 4026^{a}\end{array}$ & $\mathrm{H}$-lines well visible & H-lines visible \\
\hline B 3 & \multicolumn{2}{|c|}{$\mathrm{H}$-lines predominating in spectrum ${ }^{\mathrm{a}}$} & H-lines visible \\
\hline B 5 & \multicolumn{2}{|c|}{ Sil $4128-30$ equal to $\mathrm{He}$ I $4144^{a}$} & $\mathrm{H}$-lines dominate \\
\hline B $6-8$ & \multicolumn{3}{|c|}{ Interpolation between B9 and B5 by means of other spectra on the plate } \\
\hline B 9 & \multicolumn{3}{|c|}{ Extremely wide H-lines. No K-line visible ${ }^{\mathrm{a}}$} \\
\hline A 0 & \multicolumn{3}{|c|}{ Extremely wide $\mathrm{H}$-lines. Faint $\mathrm{K}$-line visible } \\
\hline
\end{tabular}

a Under ideal conditions according to details given in 'An atlas of Stellar Spectra' (Morgan et al., 1943). 
In cases of uncertainty the range of tolerance is directly indicated in the catalogue, for instance O5-9, B7-8 etc. Sometimes the type indication OB is used to cover the range O5-B3.

To illustrate different spectral types we have prepared reproductions of some representative spectra (Figure 1). Of course under- and overexposed spectra increase the uncertainty considerably but the most irrational sources of errors and uncertainty are connected with overlaps on the plate. When the blue end of the spectrum is disturbed by a neighbouring spectrum the presence or absence of a K-line cannot be checked.
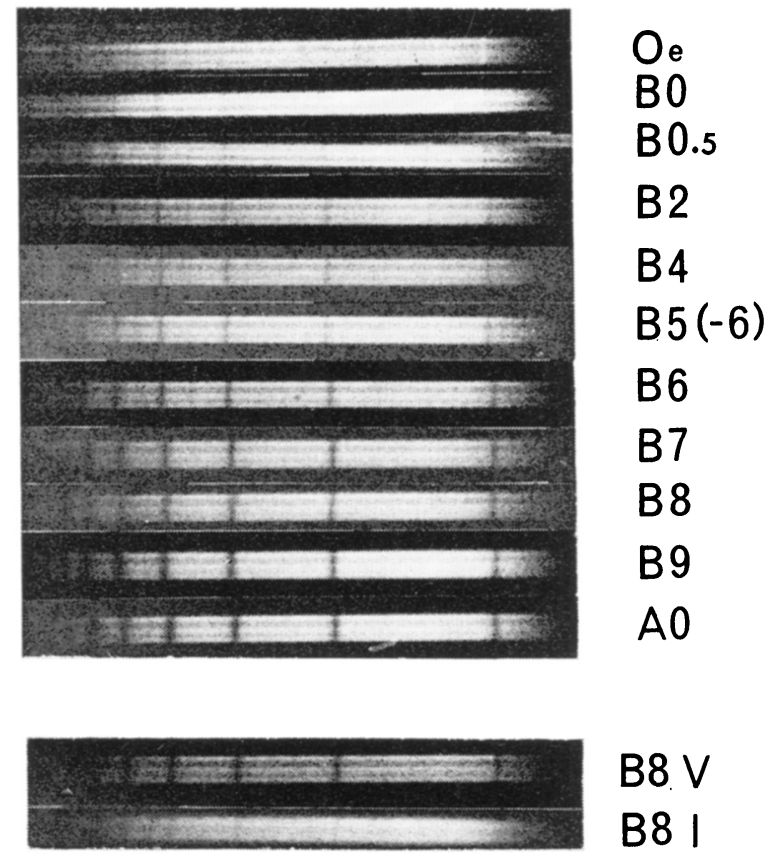

Fig. 1. The spectral types indicated refer to the present Stockholm classification.

In the case of high quality of the exposure this will not imply any serious complication, but if the He lines are blurred out, the classification becomes uncertain, which is indicated in the catalogue. There is also some risk that a composite spectrum is formed by two overlapping stars. Under unfavourable conditions such a composite spectrum may look quite similar to that of an early-type star, but 'false' objects of this kind can be expected to occur very seldom in the catalogue $(<1 \%)$.

With consideration to all sources of errors we may conclude that a certain spectral type designation in the present system does not uniquely (point-to-point) correspond to the same designation in the MK system although the deviation in an overwhelming majority of cases is rather unimportant as far as the purpose of the catalogue is 
concerned. The scheme presented in Table II will indicate the reasonable borders of uncertainty for each spectral type designation in the present system. Each spectrum has been classified by two persons one of which always has been L. O. Lodén.

\subsection{SPECTRA}

The $\mathrm{M}$ spectra are identified and classified only from the TiO bands. For the faintest stars of this type, the continuum vestiges between the bands are the only traces visible on the plate. Spectra of high quality with hardly visible TiO bands are classi-

TABLE II

Reasonable misclassification limits for the spectral types given in the Stockholm catalogues

\begin{tabular}{lll}
\hline $\begin{array}{l}\text { Spectral type desig- } \\
\text { nation }\end{array}$ & Earliest MK type & Latest MK type \\
\hline & & \\
O5, O6 & O5 & B0V or B2I \\
O7, O8 & O5 & B0V or B3I \\
O9 & O6 & B1V or B5I \\
B0 & O9 & B2V or B6I \\
B1 & B0V & B3V or B6I \\
B2 & B1V & B5V or B8I \\
B3 & B2V & B5V or B8II \\
B4 & B3V & B6V or B8II \\
B5 & B5V & B7V or B8 III \\
B6 & B5V & B8V-III \\
B7 & B6V & B9V-III \\
B8 & B7V & A0V \\
B9 & B8V & A1V \\
A0 & B9V & A2V \\
& & \\
\hline
\end{tabular}

fied as M0 and those with very well pronounced bands as M5. Other subtypes are obtained by means of interpolation and extrapolation. A considerable fraction of the M0 spectra will disappear into the late $\mathrm{K}$ ones and escape detection. For spectra later than M0 there is generally no detection problem, but it has neither been possible to establish any connection with MK-class on an absolute basis nor to distinguish between main sequence and above-main-sequence $M$ stars.

\subsection{OTHER SPECTRAL TYPES}

No attempt has been made to subclassify the carbon or S stars because of their rarity which prevents comparison between spectra on the same plate. Spectra of early or late type with evident emission lines superimposed are classified as accurately as possible although the emission lines will generally increase the uncertainty. Therefore, the designations $\mathrm{OBe}, \mathrm{Be}$, and $\mathrm{Me}$ are rather common in the catalogue. In a number of cases, only the emission lines are visible on the plates. These objects are indicated 
with an $E$ in the catalogue. The spectra of this type might represent any type of emission line stars or nebulous objects. However, certain Be stars with Balmer lines completely filled in by emission may have been mistaken for $\mathrm{O}$ stars.

\subsection{THE LIMITING MAGNITUDES}

There are at least three exposures of each partial region of the Milky Way with different exposure times, generally $1 \mathrm{~h}, 15 \mathrm{~min}$ and $4 \mathrm{~min}$. The corresponding ultimate photographic magnitude limits for a star of spectral type A should roughly be of the order of $15^{m}, 14^{m}$ and $13^{m}$ respectively. However, the practical limit is set earlier and mainly conditioned by other circumstances as for instance the state of overlap on the plate and the distortion of the spectra under extreme exposure conditions. Concerning the risk for overlap, it is important to note that the whole project is devoted to extremely star-rich regions of the Milky Way. Therefore, over a certain exposure time that will differ slightly from one region to another, one does not obtain any additional information. The faintest spectra will interfere with those over and well-exposed preventing successful classification. However, as mentioned above, the registration is restricted to spectra with outstanding appearance on the plate, and when severely underexposed most of these spectra will lose their typical features. An underexposed spectrum of type B5 will look more or less like an A0 judged from the Balmer lines and generally escape detection. As a reasonable mean value of the practical limiting magnitude for the O-B9 stars we may indicate $m_{B}=12.0$. In the case of late-type stars the situation is quite different. The molecular band features are easy to detect even if the spectrum is extremely underexposed or overlapped. Thus about $85 \%$ of the late type spectra can be detected down to $m_{B}=15.5$. For natural reasons no limiting magnitude can be indicated for the emission line objects.

\subsection{OTHER CONCEIVABLE SYSTEMS}

It might be considered whether the classification system which has been applied might be favourably replaced by a less detailed one in order to provide less ambiguous information. The system nearest at hand would then be the rather common one that includes only three principal types, $\mathrm{OB}^{+}, \mathrm{OB}$, and $\mathrm{OB}^{-}$. Careful comparisons have shown, however, that a direct classification in this system will give very ambiguous and indistinct border regions between the types and make the catalogue less useful. There are also appreciable deviations between various catalogues applying the 'OB system'. In spite of that, the 'OB system' or 'system of natural groups' is preferred by several observers because of its simplicity. It directly tells the probable actual luminosity of the star, i.e. the $\mathrm{OB}^{+}$stars are either of very early spectral type or of intermediate type and high luminosity. In many cases it is of greatest interest just to select the luminous stars for further investigations. Therefore, we have a programme for translation from the present system to the 'OB' one. Such a translation is easy to perform without introduction of too much uncertainty in the border regions. However it is not reversible which is the main reason for us to maintain our detailed system unchanged. 


\section{The Card Catalogue}

All information about the stars is stored originally on punch cards and transferred to magnetic tape and a computer disc memory. Each record (card) gives identification number, coordinates (rectangular, equatorial, galactic), estimated magnitudes (generally $m_{B}$, occasionally complete $U B V$ photometry), spectral type and sometimes

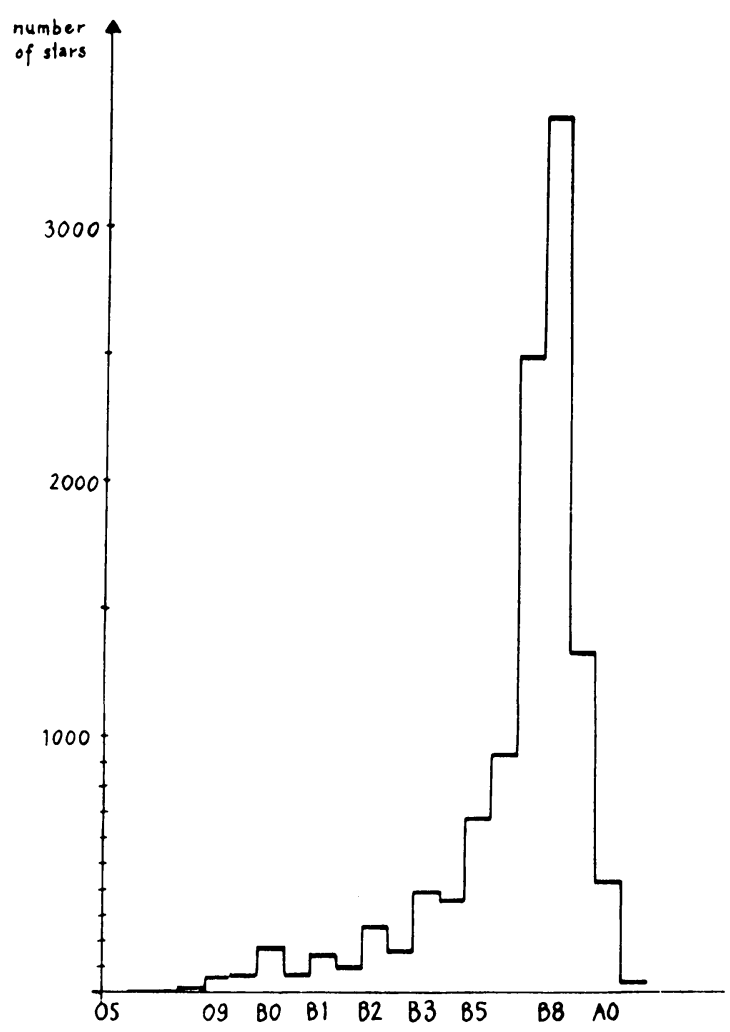

Fig. 2. The distribution of early spectral types appearing in the catalogue covering the CarinaCentaurus region $\left(l=280^{\circ}-318^{\circ}\right)$.

additional information concerning classification - quality of the spectrum, overlap, or other remarks.

Altogether we have registered 13000 stars of which 2000 are late type stars (Figure 2).

\section{References}

Lodén, L. O. and Sundman, A.: 1966, Stockholms Obs. Ann. 22, No. 10.

Morgan, W. W., Kennan, P. C., and Kellman, E.: 1943, An Atlas of Stellar Spectra with an Outline of Spectral Classification, Chicago.

Nordström, B.: 1970, List of 3000 Stars of Spectral Type O-B5 in the Galactic Region II $^{\mathrm{II}}=236^{\circ}$ to $l^{\mathrm{II}}=318^{\circ}$, unpublished.

Sundman, A., A Catalogue of $3000 O B, B$ and $M$ Stars in Centaurus, unpublished. 